\title{
MACHINE LEARNING
}

\section{Vapnik-Chervonenkis (VC) Dimension}

\author{
Alessandro Moschitti
}

Department of Information Engineering and Computer Science

University of Trento

Email: moschitti@disi.unitn.it 


\section{Computational Learning Theory}

- The approach used in rectangular hypotheses is just one simple case:

E Medium-built people

E No general rule has been derived

- Is there any means to determine if a function is PAC learnable and derive the right bound?

- The answer is yes and it is based on theVapnikChervonenkis dimension (VC-dimension, [Vapnik 95]) 


\section{VC-Dimension definition (1)}

- Def.1: (set shattering): a subset $\mathrm{S}$ of instances of a set $\mathrm{X}$ is shattered by a collection of function $F$ if $\forall \mathrm{S}^{\prime} \subseteq \mathrm{S}$ there is a function $f \in F$ such data: $f(x)= \begin{cases}1 & x \in S^{\prime} \\ 0 & x \in S-S^{\prime}\end{cases}$

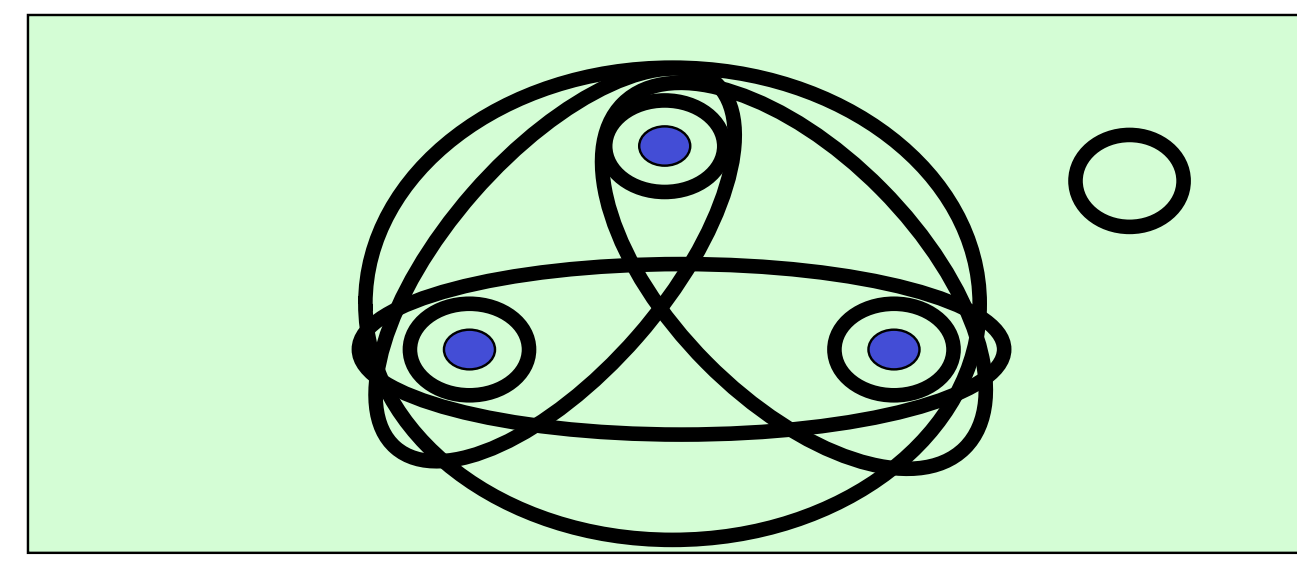




\section{VC-Dimension definition (2)}

- Def. 2: the VC-dimension of a function set F (VC$\operatorname{dim}(F))$ is the cardinality of the largest dataset that can be shattered by $F$

- Observation: the type of the functions used for shattering data determines the VC-dim 


\section{VC-Dim of linear functions (hyperplane)}

- In the plane (hyperplane = line):

- $\mathrm{VC}$ (Hyperplanes) is at least 3

- $\mathrm{VC}$ (Hyperplanes) $<4$ since there is no set of 4 points, which can be shattered by a line.

$\Rightarrow \mathrm{VC}(\mathrm{H})=3$. In general, for a $\mathrm{k}$-dimension space $\mathrm{VC}(\mathrm{H})=\mathrm{k}+1$

- NB: It is useless selecting a set of linearly independent points
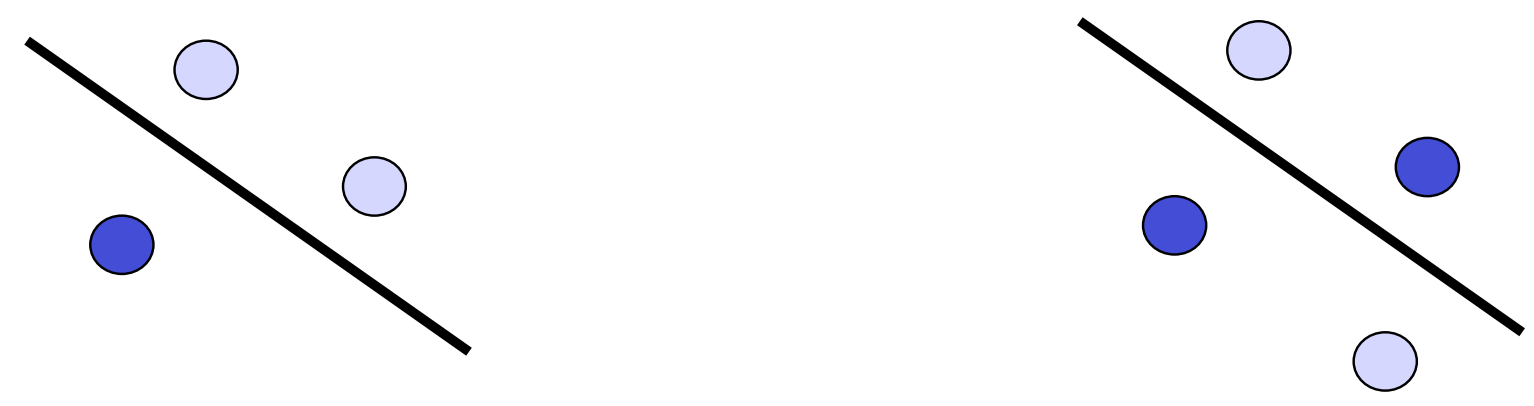


\section{Upper Bound on Sample Complexity}

Theorem 2.9 (upper bound on sample complexity, [Blumer et al., 1989]) Let $H$ and $F$ be two function classes such that $F \subseteq H$ and let $A$ an algorithm that derives a function $h \in H$ consistent with $m$ training examples. Then, $\exists c_{0}$ such that $\forall f \in F, \forall D$ distribution, $\forall \epsilon>0$ and $\delta<1$ if

$$
m>\frac{c_{0}}{\epsilon}\left(V C(H) \times \ln \frac{1}{\epsilon}+\frac{1}{\delta}\right)
$$

then with a probability $1-\delta$,

$$
\operatorname{error}_{D}(h) \leq \epsilon,
$$

where $V C(H)$ is the $V C$ dimension of $H$ and error $_{D}(h)$ is the error of $h$ according to the data distribution $D$. 


\section{Lower Bound on Sample Complexity}

Theorem 2.10 (lower bound on sample complexity, [Blumer et al., 1989]) To learn a concept class $F$ whose VC-dimension is $d$, any PAC algorithm requires $m=\Omega((d(H)+\ln (1 / \delta)) / \epsilon)$ 


\section{Bound on the Classification error using VC-dimension}

Theorem 2.11 (Vapnik and Chervonenkis, [Vapnik, 1995])

Let $H$ be a hypothesis space having $V C$ dimension $d$. For any probability distribution $D$ on $X \times\{-1,1\}$, with probability $1-\delta$ over $m$ random examples $S$, any hypothesis $h \in H$ that is consistent with $S$ has error no more than

$$
\operatorname{error}(h) \leq \epsilon(m, H, \delta)=\frac{2}{m}\left(d \times \ln \frac{2 e \times m}{d}+\ln \frac{2}{\delta}\right),
$$

provided that $d \leq m$ and $m \geq 2 / \epsilon$. 


\section{Example: Rectangles for learning medium- built person concept have $\mathrm{VC}$-dim $>4$}

- We must choose 4-point set, which can be shattered in all possible ways

- Given such 4 points, we assign them the $\{+,-\}$ labels, in all possible ways.

- For each labeling it must exist a rectangle which produces such assignment, i.e. such classification 


\section{Example (cont'd)}

- Our classifier: inside the rectangle positive and outside negative examples, respectively

- Given 4 points (linearly independent), we have the following assignments:

a) All points are "+" $\Rightarrow$ use a rectangle that includes them

b) All points are "-"” $\Rightarrow$ use a empty rectangle

c) 3 points "-" and 1 "+" $\Rightarrow$ use a rectangle centered on the "+" points 


\section{Example (cont'd)}

d) 3 points "+" and one "." $\Rightarrow$ we can always find a rectangle which excludes the "-" points

e) 2 points "+" and 2 points "-" $\Rightarrow$ we can define a rectangle which includes the 2 "+" and excludes the 2 "-."

- To show d) and e) we should check all possibilities 


\section{For example, to prove e)}

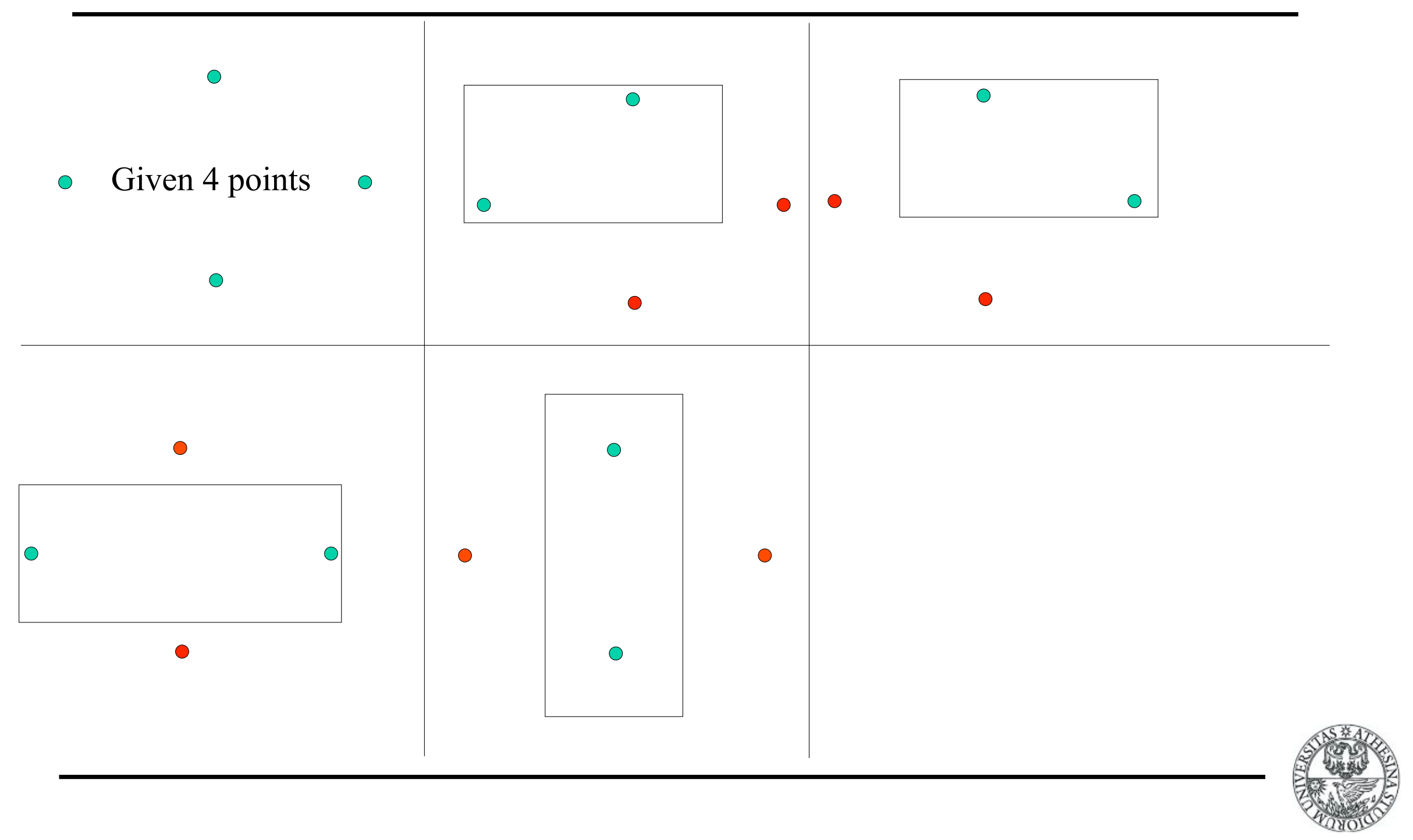




\section{VC-dim cannot be 5}

- For any 5-point set, we can define a rectangle which has the most extern points as vertices

- If we assign to such vertices the "+" label and to the internal point the "-" label, there will not be any rectangle which reproduces such assigment 


\section{Applying general lower bound to rectangles}

Theorem 2.10 (lower bound on sample complexity, [Blumer et al., 1989])

To learn a concept class $F$ whose VC-dimension is d, any PAC algorithm requires $m=\Omega((d(H)+\ln (1 / \delta)) / \epsilon)$

- $m=\mathrm{O}((4+\ln (1 / \delta)) / \varepsilon))$ 


\section{Bound Comparison (lower bound)}

- $m>(4 / \varepsilon) \cdot \ln (4 / \delta) \quad($ ad hoc bound $)$

- $m=\mathrm{O}((1 / \varepsilon) \cdot(\ln (1 / \delta)+4))=($ lower bound based on VC-dim $)$

- Does the ad hoc bound satisfy the general bound?

- $(4 / \varepsilon) \cdot \ln (4 / \delta)>(1 / \varepsilon) \cdot(\ln (1 / \delta)+4)$

$\Leftrightarrow \ln (4 / \delta)>\ln (1 / \delta) / 4+1 \Leftrightarrow \ln (1 / \delta)+\ln (4)>\ln (1 / \delta) / 4+1$

$\Leftrightarrow \ln (4)>(-1+1 / 4) \ln (1 / \delta)+1 \Leftarrow \ln (4)>1$

$\Leftrightarrow \ln (4)>\ln (\mathrm{e})$ 


\section{References}

- VC-dimension:

- MY SLIDES: http://disi.unitn.it/moschitti/ teaching.html

E MY BOOK:

- Automatic text categorization: from information retrieval to support vector learning

- Roberto Basili and Alessandro Moschitti 


\section{References}

- A tutorial on Support Vector Machines for Pattern Recognition

- Downlodable from the web

- The Vapnik-Chervonenkis Dimension and the Learning Capability of Neural Nets

- Downlodable from the web

- Computational Learning Theory

(Sally A Goldman Washington University St. Louis Missouri)

- Downlodable from the web

- AN INTRODUCTION TO SUPPORT VECTOR MACHINES (and other kernel-based learning methods)

N. Cristianini and J. Shawe-Taylor Cambridge University Press

E You can buy it also on line 


\section{Other Web References}

- On the sample complexity of PAC learning half spaces against the uniform distribution, Philip M. Long.

- A General Lower Bound on the Number of Examples Needed for Learning, Andrzej Ehrenfeucht, David Haussler, Michael Kearns and Leslie Valiant

- BOUNDS ON THE NUMBER OF EXAMPLES NEEDED FOR LEARNING FUNCTIONS, Hans Ulrich Simon

- Learnability and the Vapnik-Chervonenkis Dimension, ANSELM BLUMER, ANDRZEJ EHRENFEUCHT, DAVID HAUSSLER AND MANFRED K. WARMUTH

- A Preliminary PAC Analysis of Theory Revision, Raymond J. Mooney

- The Upper Bounds of Sample Complexity, http://mathsci.kaist.ac.kr/ nipl/ am621/lecturenotes.html 


\section{Proposed Exercises}

- Try to formulate the concept medium-built people with squares instead of rectangles and apply the content of the PAC learning lecture to this new class of functions.

- Could you build a better ad-hoc bound than the one we evaluated in class? (assume that the concept to learn is a square and not a rectangle) 


\section{Propose Exercises}

- Evaluate the VC-dimension (of course in a plane) for

E squares

E circles

- equilateral triangles

E Sketch the proof of $\mathrm{VC}<\mathrm{k}$ but do not spend to much time in formalizing such proof.

- Compare the lower-bound to the sample complexity using squares (calculated with VC dimension) with your ad hoc bound derived from medium-built people (as we did it in class for rectangles). 